\title{
Terapia della eiaculazione precoce
}

\author{
Andrea Sansone $^{1} \cdot$ Francesco Pallotti $^{1} \cdot$ Francesco Romanelli $^{1} \cdot$ Francesco Lombardo $^{1}$
}

C Springer International Publishing AG 2017

L'eiaculazione precoce (EP) è il più frequente disturbo sessuale riferito nel maschio, ed è a tutti gli effetti un sintomo auto-identificato, auto-riportato e auto-valutato da chi ne soffre. Attualmente la International Society for Sexual Medicine ha definito l'EP come un'eiaculazione che avviene sempre o quasi sempre entro 1 minuto (EP life-long) o 3 minuti (EP acquisita) dalla penetrazione, con incapacità di ritardare l'eiaculazione e conseguenze negative sul piano personale come frustrazione, sofferenza e/o condotte di rifiuto dell'atto sessuale [1]. Dal punto di vista tassonomico, possiamo riconoscere forme assolute e relazionali, organiche e psicogene (o meglio non-organiche), acquisite o life-long.

Il corretto approccio all'EP consiste nel non considerarla esclusivamente una patologia a sé stante, ma un possibile sintomo di una condizione latente $[2,3]$.

Misure di prevenzione. Valgono come utili misure preventive tutte le strategie volte a limitare la comparsa di condizioni che vedano l'EP come sintomo, come ad esempio la tireotossicosi e le infezioni del tratto genito-urinario come le prostatiti.

Procedure clinico-diagnostiche. La diagnosi di EP è come detto auto-riportata: questionari validati (PEDT: Premature Ejaculation Diagnostic Tool; PEP: Premature Ejaculation Profile) contribuiscono a facilitare la diagnosi che comunque richiede un attento colloquio clinico, volto ad investigare altri eventuali sintomi sessuali (in particolare la

$凶$ F. Lombardo

francesco.lombardo@uniroma1.it

F. Romanelli

francesco.romanelli@uniroma1.it

1 Dipartimento di Medicina Sperimentale, Sezione di Fisiopatologia Medica, Endocrinologia e Scienza dell' Alimentazione, "Sapienza” Università di Roma, Roma, Italia disfunzione erettile) e fattori psicologici. Identificare eventuali cause psicogene o organiche non esclude tuttavia la compresenza di entrambe.

Procedure terapeutiche. Ove l'EP sia sintomo di una condizione morbosa, come ad esempio l'ipertiroidismo o uno stato irritativo del tratto genito-urinario, la terapia deve essere finalizzata alla risoluzione della suddetta patologia. Qualora l'EP resti invece senza una causa chiaramente identificabile, trovano indicazione terapie non-farmacologiche e farmacologiche. Le prime includono il counseling, utile soprattutto nei casi di eccessiva ansia da prestazione, ma anche le tecniche dello squeeze e dello stop-and-start, che consistono rispettivamente nella costrizione manuale del glande e nell'interruzione del rapporto al fine di ritardare l'eiaculazione. La terapia farmacologica con inibitori della fosfodiesterasi di tipo 5 (sildenafil, vardenafil, tadalafil e avanafil) trova specifica indicazione nelle condizioni in cui l'EP sia associata a disfunzione erettile: la maggiore efficacia nel ritardare l'eiaculazione è stata documentata soprattutto per il vardenafil. Paroxetina, sertralina e fluoxetina, inibitori selettivi del reuptake della serotonina (SSRI), sono stati utilizzati fuori scheda tecnica con significativi problemi di efficacia e tollerabilità, particolarmente nell'uso quotidiano; la dapoxetina è ad oggi l'unico farmaco appartenente a questa classe progettato specificatamente per la terapia on demand dell'EP, con adeguati e documentati parametri di efficacia. Una menzione meritano anche gli anestetici locali, in primis la lidocaina e la prilocaina, usati off-label per ridurre la sensibilità peniena (Tabella 1).

Procedure riabilitative. L'ansia da prestazione, non più fenomeno caratteristico del giovane, trova spesso giovamento dalla terapia (farmacologica e non) dell'EP; viceversa, in molti casi la terapia comportamentale e l'approccio psico- 
Tabella 1 Schema riassuntivo delle principali terapie per la EP

\begin{tabular}{|c|c|c|c|c|}
\hline $\begin{array}{l}\text { Classe } \\
\text { farmacologica }\end{array}$ & Principio attivo & Formulazione & Posologia & Indicazioni \\
\hline $\begin{array}{l}\text { Inibitori delle } \\
\text { fosfodiesterasi } \\
\text { tipo } 5 \text { (PDE5i) }\end{array}$ & $\begin{array}{l}\text { Sildenafil } \\
\text { Vardenafil } \\
\text { Tadalafil } \\
\text { Avanafil }\end{array}$ & $\begin{array}{l}\text { Compresse/ } \\
\text { Compresse } \\
\text { orodispersibili }\end{array}$ & $\begin{array}{l}\text { Al bisogno o in } \\
\text { cronico }\end{array}$ & $\begin{array}{l}\text { EP con } \\
\text { concomitante DE }\end{array}$ \\
\hline $\begin{array}{l}\text { Inibitore selettivo } \\
\text { del reuptake della } \\
\text { serotonina (SSRI) }\end{array}$ & Dapoxetina & Compresse & Al bisogno & $\begin{array}{l}\text { Unico farmaco } \\
\text { approvato per EP }\end{array}$ \\
\hline $\begin{array}{l}\text { Inibitori selettivi } \\
\text { del reuptake della } \\
\text { serotonina (SSRI) }\end{array}$ & $\begin{array}{l}\text { Paroxetina } \\
\text { Sertralina } \\
\text { Fluoxetina }\end{array}$ & Compresse & $\begin{array}{l}\text { Al bisogno o in } \\
\text { cronico }\end{array}$ & Off label \\
\hline Anestetici topici & $\begin{array}{l}\text { Lidocaina } \\
\text { Prilocaina }\end{array}$ & Crema/Pomata & Al bisogno & Off label \\
\hline
\end{tabular}

*Uso cronico riconosciuto solo per tadalafil $5 \mathrm{mg}$

sessuologico rappresentano utili meccanismi per prevenire, o curare, i diversi sintomi sessuali.

Conflitto di interesse Gli Autori dichiarano di non avere conflitti di interesse.

Consenso informato Lo studio presentato in questo articolo non ha richiesto sperimentazione umana.

Studi su animali Gli Autori di questo articolo non hanno eseguito studi sugli animali.

\section{Bibliografia}

1. Althof SE, McMahon CG, Waldinger MD et al (2014) An update of the International Society of Sexual Medicine's guidelines for the diagnosis and treatment of premature ejaculation (PE). J Sex Med 11:1392-1422

2. Althof SE, McMahon CG (2016) Contemporary management of disorders of male orgasm and ejaculation. Urology 93:9-21

3. Jannini EA, Lenzi A (2015) Inquadramento diagnostico e terapia medica dell' eiaculazione precoce. L'Endocrinologo 16:253-257 